\title{
New Methodology for Translation of Static Sign Symbol to Words in Kannada Language
}

\author{
Ramesh M. Kagalkar \\ Research scholar VTU, Belgaum, \\ Karnataka, India
}

\author{
Nagaraj H.N, PhD \\ Professor and Director, IITE, \\ Ahmedabad, Gujarath, India
}

\begin{abstract}
Communication is the mean for data transfer over a medium to interact their expressions with each other. The common mode of communication is vocal speech conversation. The main modes of communication are constraint to vocally disabled individuals. For the communication of such individuals various means of communication is suggested, which are called as sign language. Aim: The aim of sign language alphabets recognition is to provide an easy, efficient and accurate mechanism for automatic translation of static sign (determined by a certain configuration of hand) to textual version in kannada language. Problem Statement: The work presented in this paper goal to develop a system for automatic translation of static gestures of alphabets in kannada sign language. It maps letters, words and expression of a certain language to a set of hand gestures enabling an in individual to communicate by using hands gestures rather than by speaking. The system capable of recognizing sign language symbols can be used as a means of communication with hard of hearing people. Sign of the deaf individual can be captured, recognized and translated to words in kannada language for the benefit of blind people. Approach: It has been divided into two phases firstly, feature extraction phase which in turn uses histogram technique, Hough and Segmentation to extract hand from the static sign. Secondly classification phase uses neural network for training samples. Extreme points were extracted from the segmented hand using star skeletonization and recognition was performed by distance signature. Results: The proposed method was tested on the dataset captured in the closed environment with the assumption that the user should be in the field of view. This study was performed for five different datasets in varying lighting conditions. Conclusion: The developed system is focused with objective of reducing the communication gap between normal people and vocally disabled.
\end{abstract}

\section{Keywords}

Kannada Sign Language KSL, Hearing-Impaired HI.

\section{INTRODUCTION}

Humans understand one another by convincing their ideas, thoughts, and experiences to the people around them. There area unit varied ways that to realize this and therefore the best one of the remainder is the gift of speech. Through speech everybody will terribly convincingly transfers their thoughts and perceive one another. It will be injustice, if we have a tendency to ignore people who area unit empty this valuable gift. The sole suggests that of communication on the market to the vocally disabled is that the use of sign language communication. The exploitation sign language communication they're restricted to their own world. This limitation prevents them from interacting with the outer world to share their feelings, artistic ideas and potentials. Only a few people that do not seem to be themselves deaf ever learn to sign language communication. This limitation will increase the isolation of deaf and dumb people from the common society. Technology is a method to get rid of this hindrance and profit these people many researchers have explored these prospects and have with success achieved finger spelling recognition with high levels of accuracy. However progress within the recognition of sign language communication, as a full has varied limitations in today's applications. Varied systems were projected for the automated recognition of sign language communication.

\section{SIGN LANGUAGE}

Sign language could be a visual language consisting of varied signs, gestures, finger spelling and facial expressions. It's the foremost common and natural suggests that of communication for the Hearing Impaired (HI). Historically, world's deaf community has been in a disadvantage position within the society thanks to their inability to listen to, speak and properly communicate with others, let alone the indifference of the remainder of the people to be told signing employed by HI persons to specific their mind. Hence, there arises the requirement to develop some helpful tools, which might give an interface between a sign language and its spoken counterpart, in order that the gap between the two segments of the society may be decreased. Speech could be a generic mode of communication to specific the thinking from one individual to different. In varied eventualities, there may well be a limitation in providing communication between people and disabled persons. During this sort of things signing is one mode of communication for them. Hearing impaired will communicate through sign with people. Typically the sign language is additionally tough for the people to own communication some could perceive their views some might not. Therefore there is a necessity for one system that lays a bridge between them with none problem in establishing communication.

During this work an automatic system is projected, wherever a cue image is taken as input and a text are going to be an output. If it is the case, anyone will perceive what people are attempting to specific. In recent year the analysis has progressed steady in relevancy the utilization of computers to acknowledge and render signing. The sign are not international countries have distinctive sign. Deaf people need to be treated same as people with equal rights and revered. Deaf people with skills data and valuable experiences, which might be shared like traditional people. There is being such a large amount of organizations that have return front, so as to scale back the 
matter of deaf people. Among them Akshay akruti is one. Akshay akruti could be a registered, non-profit, non-religious organization operating for hearing improved children's. It's established in Hyderabad, Andhra Pradesh in 1996. At first this organization started with five childerns and currently to the tutorial wants of 163 children's. The most feature of this organization is being that the kids are inspired to talk through and actual methodology of teaching. The main target is to reinforce the residual hearing powers of the kids. So encouraging the kids to talk instead of deaf on sign communication.

Deaf people face some challenges that the hearing world doesn't face and will notice troublesome to grasp deaf people face challenges. Deaf drivers should specialize in their attention, once driving associate automobile as a result of the are not capable to listen to traffic noise. Even the handicapped persons have support of family and friends; it's doubtless that they're the sole deaf person within the family one cluster of friends. This could lead some deaf people to feel alone within the world. There is another downside that the deaf people facing. They are within the education field and albeit, the deaf people have the talent however he is not given preference. It additionally one among the downside for the deaf people. As deaf people are not getting tired to elucidate, the implication of hearing impairment is as linguistic and cultural distinction between deaf and hearing persons: Hearing impairment implies a specific approach of individuals developing their communication functions and there with return different variations. Moreover, communication in sign languages implies specific ways that of coping with social interactions, so deaf people naturally organize deaf communities at intervals the hearing societies during which they live and naturally build a culture of their own.

\section{LITERATURE SURVEY}

For the popularity of the signing slightly screen based mostly approach is developed in [1]. The author tries to acknowledge the character generated from the screen device and rework to speech signal supported a recognition algorithmic program. In an approach [2] the author suggests in recognizing the hand gesture supported the finger boundary tracing and tip detection. The author urged to spot the kannada sign supported the hand gesture passed. In [3] a computing approach at hand gesture recognition is developed for hearing and speech impaired. Don Pearson in his approach communication systems for the deaf [4], conferred a two manner communication approach, wherever he planned the usefulness of

switched TV for each deaf to hearing and deaf to deaf communication. In his approach, attention is given to the necessities of image communication

systems that modify the inattentive communicate

over distances victimization phone lines. The visual language of the deaf is formed from signs [5], that area unit gestures created primarily with the hands and arms, and conjointly with the face and different components of the body. Sign will convey sophisticated ideas that a standard person uses sentences. Sentences area unit at a rate that is concerning identical as for a speech. The speed at that sentences area unit delivered victimization finger-spelling slows down, as additional words ought to be spelled during this manner. Signing, finger writing system, and lip-reading area unit ordinarily used for communication between deaf individuals and customary individuals. Finger-spelling is employed for technical words, place names, etc., wherever every letter needs to be communicated victimization fingers. Jesus F. Guitarte Perez, Alejandro F. Frangi, Eduardo Lleida Solano, and Klaus Lukas in their paper conferred a lip reading technique to boost the popularity rate on embedded setting [6]. They compare the performance of lip reading and therefore the standard noise reduction systems in degraded eventualities. They conferred an approach to use the improved speech recognition algorithmic program for deaf and dumb application. Towards the event of machine-driven speech recognition for vocally disabled individuals a system referred to as Boltay Haath [7] is developed to acknowledge West Pakistan signing (PSL) at Sir Syed university of engineering and technology. The Boltay Haath project aims to provide sound matching the accent and pronunciation of the individuals from the sign image passed. A carrying glove for vocally disabled is intended, to remodel the signed symbols to sounding speech signals victimization gesture recognition. They use the movements of the hand and fingers with sensors to interface with the pc. The system ready to eliminate a serious communication gap between the vocally disabled with common community. However Boltay Haath has the limitation of reading solely the hand or finger movements neglecting the body action, that is additionally wont to convey message. This offers a limitation to solely reword the finger and palm movements for speech transformation. The opposite limitation which will be seen with Boltay Haath system is that the signer may well be ready to communicate with a standard person however the contrariwise is not doable with it. This offers the limitation of unidirectional communication between the listeners and vocally disabled. A system is planned to beat the limitation of Boltay Haath system and supplya communication link from traditional person to physically disabled person.

\section{SYSTEM DESIGN AND IMPLEMENTATION}

The system is designed to visually recognize all static signs of the Kannada Sign Language (KSL) and all signs of alphabets using bare hands. The user/signers are not required to wear any gloves or to use any devices to interact with the system. But, since different signers vary their hand shape size, body size, operation habit and so on, which bring more difficulties in recognition. Therefore, it realizes the necessity for signer independent sign language recognition to improve the system robustness and practicability in the future. The system gives the comparison of the three feature extraction methods used for KSL recognition and suggest a method based on recognition rate. It relies on presenting the gesture as a feature vector that is translation, rotation and scale invariant. The combination of the feature extraction method with excellent image processing and neural networks capabilities has led to the successful development of KSL recognition system. The system has two phases: the feature extraction phase and the classification phase. Images were prepared using portable document format form so the system will deal with the images that have a uniform background. The feature extraction applied an image processing technique which involves algorithms to detect and isolate various desired portions of the digitized sign. During this phase, each colored image is resized and then converted from RGB to gray scale one. This is followed by an edge detection technique. The goal of edge detection is to mark the points in an image at which the 
intensity changes sharply. Sharp changes in image properties usually reflect important invents and changes in world properties. The next important step is the application of proper feature extraction phase and the next is the classification phase, a three-layer, feed-forward back propagation neural network is constructed.

\subsection{Feature Extraction Phase}

Images of signs were resized to 80 by 64 , by default imresize uses nearest neighbor interpolation to determine the values of pixels in the output image but other interpolation methods can be specified. Here bicubic method is used because if the specified output size is smaller than the size of the input image, imresize applies a low pass filter before interpolation to reduce aliasing. Therefore we get default filter size 11-by 11. To alleviate the problem of different lighting conditions of signs taken and the HSV (Hue, Saturation, Brightness) nonlinearity by eliminating the HSV information while retaining the luminance. The RGB color space (Red, Green and Blue which considered the primary colors of the visible light spectrum) is converted through gray scale image to a binary image. Binary images are images whose pixels have only two possible intensity values. They are normally displayed as black and white. Numerically, the two values are often 0 for black and either 1 or 255 for white. Binary images are often produced by Thresholding a gray scale or color image from the background. This conversion resulted in sharp and clear details for the image. It is seen that the RGB color space conversion to HSV color space then to a binary image produced images that lack many features of the sign. So edge detection is used to identify the parameters of a curve that best fir a set of given edge points. Edges are significant local changes of intensity in an image. Edges typically occur on the boundary between two different regions in an image. Various physical events cause intensity changes. Goal of edge detection is to produce a line drawing of a scene from an image of that scene. Also important features can be extracted from the edges. And these features can be used for recognition. Here canny edge detection technique is used because it provides the optimal edge detection solution. Canny edge detector results in a better edge detection compared to Sobel edge detector. The output of the edge detector defines where features are in the image. Canny method is better, but in some cases it provides extra details more than needed. To solve this problem a threshold of 0.25 is decided after testing different threshold values and observing results on the overall recognition system.

Feature extraction methods uses,

1. Histogram technique

2. Hough

3. OTSU's segmentation algorithm

4. Segmentation and extraction with edge detection

\subsection{Classification Phase}

The classification of neural network has 256 instances as its input vector, and 214 output neurons in the output layer classification phase includes network architecture, creating network and training the network. Network of feed forward back propagation with supervised learning is used.

\section{RESULTS ANALYSIS AND DISCUSSION}

The performance of the recognition system is evaluated by testing its ability to classify signs for both training and testing set of data. The effect of the number of inputs to the neural network is considered.

\subsection{User Input}

The user should make hand sign gestures which will be the input for the system for sign language recognition. During the training phase of the application, the user creates a data base of his /her hand sign gesture images. The training phase is complete when the system has captured enough gestures for which it knows the class and the system is then ready to recognize gestures. The gestures are captured by the webcam with a constant distance between the hand of the user and the camera. The illumination should also ideally be constant. a block color board is kept behind to make the background constant and make the hand area identification process easier.

For testing the unknown signs we have created a GUI as shown in figure 3,4 ,5 and 6 below, which provides the user an easy way to select any sign he/she wants to test and then after clicking on the apply pushbutton, it will display the meaning of the selected sign, look at below figures shows different GUI options. The figure 3 shows training of data base windows. The figure 4 shows after selecting query sample, it is being display on to the user. The figure 5 shows testing selecting query sample and it extract features from the query sample and finally the figure 6 shows recognized character from the query sample. The system is implemented in Matlab version 6.5. The recognition training and tests were run on a modern standard PC (1.5 GHz AMD processor, 128 MB of RAM running under windows 2000.) WEB-CAM-1.3 is used for image capturing.

\subsection{Data Set}

The data set used for training and testing the recognition system consists of gray scale images for all the KSL signs used in the experiments. Also 8 samples for each sign will be taken from 8 different volunteers. For each sign 5 out of 8 samples will be used for training purpose while remaining 5 signs were used for testing. The samples will be taken from different distances by web camera and with different orientations. In this way a data set will be obtained with cases that have different sizes and orientations and hence can examine the capabilities of the feature extraction scheme.

Following figure 1 and 2 shows kannada sign language and basic letter list, consider for training.

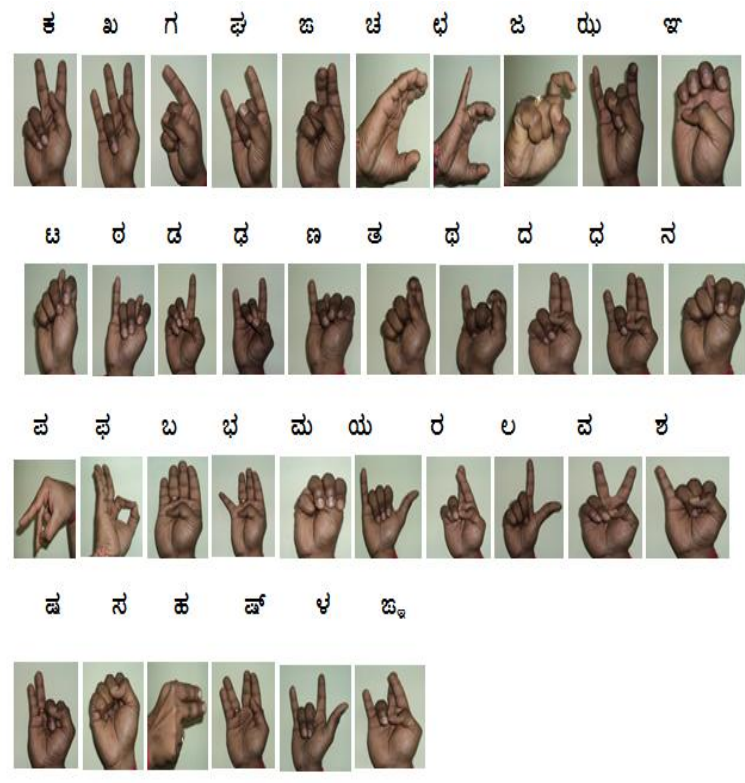

Figure 1.Shows a kannada sign symbol basic letter (16). 


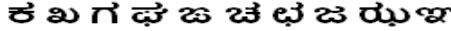

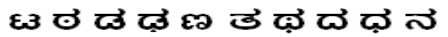

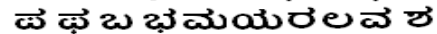

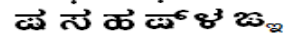

Figure 2.Shows a kannada basic 16 letters lists.

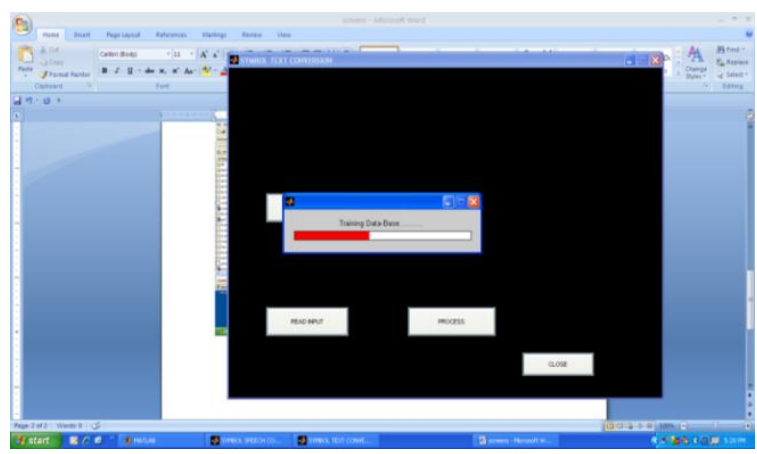

Figure 3. Shows training of data base windows.

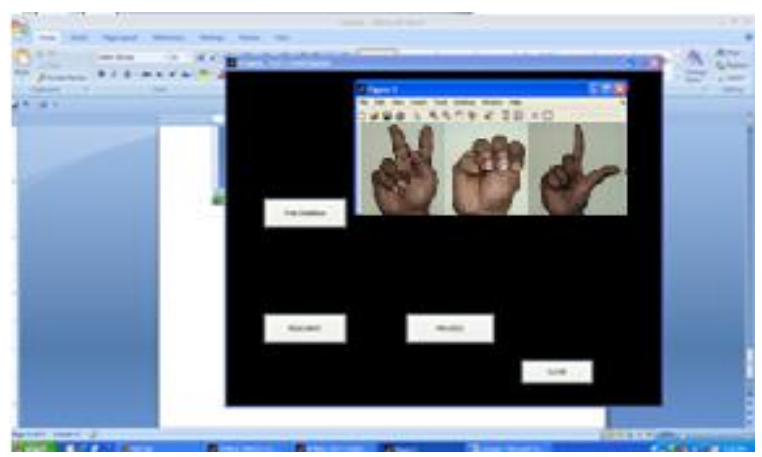

Figure 4 Shows after selecting query sample and is being display on to the user.

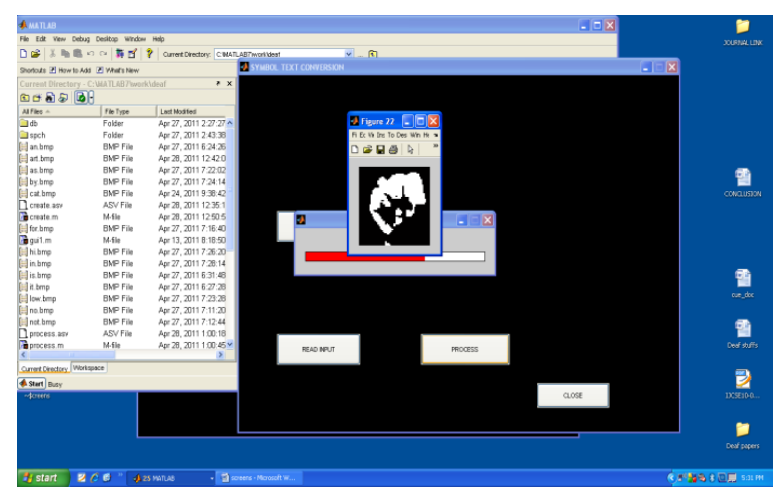

Figure 5. Shows after selecting query sample and it extract features from the query sample.

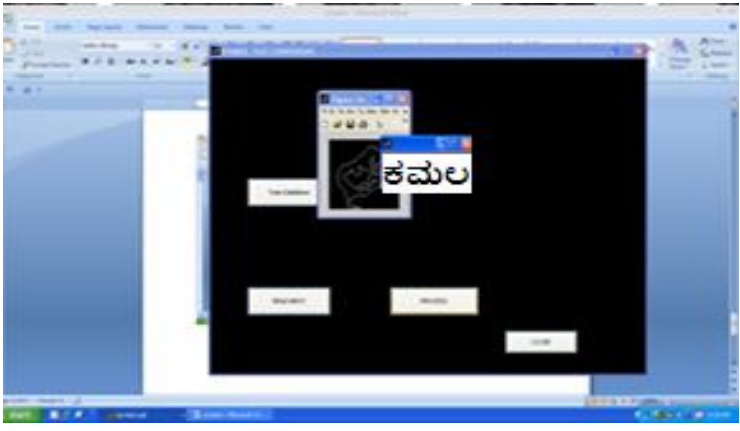

Figure 6. Shows recognized word of kannada language from the query sample.

\section{CONCLUSION}

Deaf and dumb people rely on sign language interpreters for communication. However, they cannot depend on interpreters every day in life mainly due to the high costs and the difficulty in finding and scheduling qualified interpreters. This system will help disabled persons in improving their quality of life significantly. The automatic recognition of sign language is an attractive prospect; the technology exists to make it possible, while the potential applications are exciting and worthwhile. The system is proved robust against changes in gesture. Using histogram technique we get the misclassified results. Hence histogram technique is applicable to only small set of KSL alphabets or gestures which are completely different from each other. It does not work well for the large or all 592 number of set of KSL signs. For more set of sign gestures segmentation method is suggested. The main problem with this technique is how good differentiation one can achieve. This is mainly dependent upon the images but it comes down to the algorithm as well. It may be enhanced using other image processing technique like edge detection as done in the presenting paper. We used the well-known edge detector like Canny, Sobel and Prewitt operators to detect the edges with different threshold. We get good results with Canny with 0.25 threshold value. Using edge detection along with segmentation method good recognition rate is achieved. Also the system is made background independent. As we have implemented sign to text interpreter in future reverse system can also possible to implement that is text to sign interpreter.

\section{ACKNOWLEDGMENT}

The authors would like to thank Shri Chairman D.Y.Patil and Management and the Director/Principal Dr. Uttam B. Kalwane Guide and Colleagues of the Department of Computer Engineering, Dr.D.Y.Patil School of Engineering and Technology, Charoli, B.K. Via Lohegaon, Pune, Maharashtra, India, for their support, suggestions and encouragement.

\section{REFERENCES}

[1] Jung-Bae Kim,Kwang-Hyun Park,Won_Chul Bang and Z.Zenn Bien Div. of EE,Dept of EECS,KAIST,Daejeon,Republic of Korea.Continuous Korean sign language recognition using gesture segmentation and HMM. IEEE-2010.

[2] Venkatraman.S and T.V. Padmavathi, " Speech For The Disabled" , Proceedings of the International MultiConferenceof Engineers and Computer Scientists 2009 Vol I IMECS 2009,March 18 - 20, 2009. 
[3] Gaurav N. Pradhan, Chuanjun Li, Balakrishnan Prabhakaran, "Hand Gesture-based Computing for Hearing and Speech Impaired", IEEE Multimedia Magazine, Vol. 15, No. 2, pp. 20-27, April-June 2008.

[4] Aleem khalid ,Ali M, M. Usman, S. Mumtaz, Yousuf "Bolthay Haath - Paskistan sign Language Recgnition" CSIDC 2005

[5] Kadous, Waleed "GRASP: Recognition of Australian sign language using Instrumented gloves", Australia, October 1995,pp. 1-2,4-8.

[6] D. E. Pearson and J. P. Sumner, "An experimental visual telephone system for the deaf," J . Roy. Television Society vol. 16, no. 2. pp. 6-10, 1976.

[7] Guitarte Perez, J.F.; Frangi, A.F.; Lleida Solano, E.; Lukas, K. "Lip Reading for Robust Speech Recognition on Embedded Devices" Volume 1, March 18-23, 2005 PP473 - 476 .

[8] Amit kumar and Ramesh Kagalkar " Advanced Marathi Sign Language Recognition using Computer Vision", International Journal of Computer Applications (0975 8887) Volume 118 - No. 13, May 2015.

[9] Amitkumar and Ramesh Kagalkar "Sign Langauge Recognition for Deaf User", Internal Journal for Research in Applied Science and Engineering Technology, Volume 2 Issue XII, December 2014.

[10] DONPEARSON "Visual Communication Systems for the Deaf" IEEE transactions on communications, vol. com-29, no. 12, December 1981

[11] T. Masuko, K. Tokuda, T. Kobayashi, and S. Imai, "Speech synthesis using HMMs with dynamic features,' in Proc. ZCASSP-96, May 1996, pp. 389-392.

[12] SantoshKumar,S.A.; Ramasubramanian, V." Automatic Language Identification Using Ergodic HMM" Acoustics, Speech, and Signal Processing, 2005. Proceedings. (ICASSP'05).IEEE International Conference Vol1,March18-23,2005Page(s):609-612

[13] honggang wang, ming c. leu and cemil oz, "American Sign Language recognition using multidimensional Hidden Markov Models. Journal of information science and engineering 22, 1109-1123(2006). Department of Industrial Engineering Purdue University.

[14] CULSHAW MURRY (1983) "It Will Soon Be Dark. The situation of disabled in India. Delhi lithouse publications.

[15] Deshmukh Dilip (1994) the status of sign language in deaf education in India. Signpost. Newsletter of International Sign Language Association 7(1) 49-52.

[16] Starner, T. (1995). Visual recognition of American Sign Language using hidden Markov models. Master's thesis, Massachusetts Institutes of Technology.

[17] Duda, R. O., \& Hart, P. E. (1972). Use of Hough transformation to detect lines and curves in pictures. Communications of the ACM, 15, 11-15.

[18] Kourosh Khoshelham. Extending the use of Hough Transform to detect 3D objects in laser range data. ISPRS Workshop on Laser Scanning 2007 and SilviLaser2007, Espoo, September 12- 14,2007,Finland.

[19] Ong, S., and Ranganath, S. (2005) Automatic sign language analysis: a survey and the future beyond lexical meaning. IEEE Transactions on Pattern Analysis and Machine Intelligence, 27(6).

[20] Symeoinidis, k. (2000). "Hand gesture recognition-using neural networks." Master's thesis, University of Surrey.

[21] Vamplew,p.(1996). Recognition of sign language using neural networks PhD Thesis, Department of computer Science, and University of Tasmania.

[22] Watson, R. (1993). A survey of gesture recognition techniques. Technical report TCD-CS-93-11, Department of computer Science, Trinty College Dublin.

[23] C.W. Ong and surendra and ranganath Automatic sign language analysis: A survey and future beyond lexial meaning sylvie IEEE transactions on pattern analysis and machine intelligence, Vol27,No.6,June 2006.

[24] Joe Naoum-Sawaya,Mawaya,Mazen Slim,Sami Khawam and Mohamad Adnan Al-Alaoui Dynamic system design for American Sign Language Recognition by Electrical and Computer Engineering Department American University of Beirut,Lebanon. IEEE.

[25] Real-Time American Sign Language Recognition from Video Using hidden Markov Models by Thad Starner and Alex Pentland AAAI Technical Report FS- 96-05.

[26] Ramesh.M.Kagalkar and P.N.Girija " Neural Network Based Document Image Analysis for Text, Image Localization Using Wavelet Decomposition and Mathematical Morphology" International Journal on Computer Science and Information Technology(IJCEIT) Volume 16, No 21, ISSN 0974-2034, Jan-Feb 2010.

[27] Ramesh.M.Kagalkar, Mrityunjaya .V. Latte and Basavaraj.M.Kagalkar "An Improvement In Stopping Force Level Set Based Image Segmentation" International Journal on Computer Science and Information Technology(IJCEIT) ISSN 0974-2034, Volume 24, Issue No 01, June - August 2010.

[28]. Dr.M. Siddappa and Ramesh M.Kagalkar "Methodologies for Tumor Detection Algorithm as Suspicious Region from Mammogram Images Using SVM Classifier Technique" CiiT International Journal Of Digital Image Processing Print: ISSN 0974-9691 and Online: ISSN 0974-9586, Jan-Feb 2012.

[29] Mrunmayee Patil and Ramesh Kagalkar “ An Automatic Approach for Translating Simple Images into Text Descriptions and Speech for Visually Impaired People", International Journal of Computer Applications (0975 8887) Volume 118 - No. 3, May 2015.

\section{AUTHOR PROFILE}

Ramesh. M. Kagalkar was born on Jun 1st, 1979 in Karnataka, India and presently working as a Assistant. Professor, Department of Computer Engineering, Dr.D.Y.Patil School Of Engineering and Technology, Charoli, B.K.Via Lohegaon, Pune, Maharashtra, India. He has 13.5 years of teaching experience at various institutions. He is a Research Scholar in Visveswaraiah Technological University, Belgaum, He had obtained M.Tech (CSE) Degree in 2006 from VTU Belgaum and He received BE (CSE) Degree in 2001 from Gulbarga University, Gulbarga. He is the author of text book Advance Computer Architecture which cover the syllabus of final year computer science and engineering, Visveswaraiah Technological University, Belgaum. $\mathrm{He}$ is waiting for submission of two research articles for patent right. He has 
published more than 18 research papers in International Journals and presented few of there in international conferences. His main research interest includes Image processing, Gesture recognition, speech processing, voice to sign language and CBIR. Under his guidance four ME students awarded degree in SPPU, Pune, five students at the edge of completion their ME final dissertation reports and two students started are started new research work and they have publish their research papers on International Journals and International conference.

Dr.H.N Nagaraja was born on 13 December 1963 at Hosanagara in Shimoga district in Karnataka, India and he presently working as a Professor and Director, IITE Ahmedabad, Gujarat, India. He has the vast teaching experience of 28 years at various level, since 2007 to 2011. $\mathrm{He}$ was heading SDM Institute of Technology, Ujire as principal. Since 2011 he was worked as principal and director of AITM, Belgaum, Karnataka and also worked as PG Coordinator, Department of PG studies, Visveswaraiah Technological University, Belgaum and $\mathrm{He}$ had obtained Ph.D Degree in Power Electronics from IIT, Kharagapur, India in 2006, M.E Degree from WCE Sagali, Maharastra in 1991 and received BE (E\&E) Degree in 1985 from Govt. B.D.T College of Engineering, Davanagre, Karnataka. He is the life member of many national and international societies. He has presented more than 36 papers in the national and international conference and journals. He has also given 15 invited lecturers at various engineering colleges. He has been conferred best paper presentation award in the national conference held at Vellore Institute of Technology, Vellore in the year 2003. His research papers were presented at Japan, Hongkong and Malaysia. 